\title{
Fomentando la multidisciplinariedad y el trabajo en equipo (metodologías participativas): creación de empresas, una asignatura para cuatro grados.
}

\author{
Promoting multidisciplinarity and teamwork (participating \\ methodologies): entrepreneurship, a subject in four bachelors.
}

\author{
Cristina Santandreu-Mascarell (crisanma@omp.upv.es) \\ Lourdes Canós-Darós (loucada@omp.upv.es)
}

José Marin- Roig (jomara@eln.upv.es)

Universitat Politècnica de València, Escuela Politécnica Superior de Gandía (SPAIN) http://dx.doi.org/10.12795/EDUCADE.2015.i06.06

RESUMEN: Numerosos trabajos realizados en las últimas décadas han puesto de manifiesto la utilidad de las técnicas de creatividad para mejorar la generación de ideas (Michalko, 2000; Gray 2012), valorarlas (Kim, 2008; Ries, 2012) y plasmarlas en un plan de negocio (Osterwalder, 2011; Kawasaki, 2007; Christensen, 2011). Del mismo modo, la experiencia transmitida por diversos emprendedores es muy enriquecedora para poder seguir su ejemplo y no replicar los errores que cometieron en sus principios. En esta área de trabajo los docentes del Campus de Gandia de la Universidad Politécnica de Valencia creen que es necesario aprovechar las grandes posibilidades que nos brindan actualmente estas herramientas para enriquecer los ambientes de aprendizaje y que se asemejen al ámbito profesional.

La innovación docente surge al crear una asignatura optativa común para los cuatro grados, Creación de Empresas. Su objetivo consiste en, mediante el trabajo en equipo y la utilización de técnicas de creatividad, dar lugar a un modelo de negocio innovador, actual y distinto. En esta asignatura, alumnos de diferentes grados comparten el aula y comparten proyectos de negocio, de modo que aúnan distintos puntos de vista ante la resolución de problemas.

La metodología utilizada es la participación activa y la adaptación del esquema de la creatividad de Iglesias y Rodicio (2013). Aunque los resultados obtenidos no se pueden considerar significativos por ser el primer curso, nos han permitido realizar propuestas de mejora; la principal idea es conseguir que el alumno aprenda a trabajar con metodologías a las que no están acostumbrados.

Este trabajo se centra en exponer el proceso de innovación que se ha puesto en marcha durante el curso académico 2013-2014 para los cuatro grados que se imparten en la Escuela Politécnica Superior de Gandía de la Universitat Politècnica de València. Una de las intenciones fundamentales de la apuesta por estas acciones que impulsan la innovación, la generación de nuevos negocios e ideas de negocio es la de dar salida mediante el emprendedurismo y el intraemprendedurismo; dada la difícil situación económica actual, esta es una vía de mejora de la economía también en general.

PALABRAS CLAVE: creación de empresas, emprendedurismo, intraemprendedurismo, multidisciplinaridad, creatividad, trabajo en equipo, innovación.

Innodoct 2014 special track

Artículo de investigación. Recibido: 26-09-14 - Versión revisada: 27-01-15, Aceptado: 18-04-15

Licencia Creative Commons BY NC ND · 2015 · Asociación Española de Contabilidad y Administración de Empresas - AECA 
ABSTRACT: Several studies conducted in recent decades have shown the usefulness of creativity techniques to improve the generation (Michalko, 2000; Gray 2012), valuation (Kim, 2008; Ries, 2012) and translation of ideas into a business plan (Osterwalder, 2011; Kawasaki, 2007; Christensen, 2011). Similarly, the experience transmitted by different entrepreneurs is very rewarding to follow their example and not replicate mistakes they made in their startings. In this area of work, lecturers in Gandia Campus, Universitat Politècnica de València, believe it is necessary to take advantage of the great opportunities these tools currently provide to enrich learning environments that resemble the professional field.

Teaching innovation arises from the creation of a common elective course for four bachelors, Entrepreneurship. The aim is, through teamwork and the use of creativity techniques, to generate an innovative business model. In this course, students from different bachelors share the classroom and share business projects, so that different points of view are combined to solve problems.

Used methodology is active participation and the adaptation of creativity scheme from Iglesias and Rodicio (2013). Although the results cannot be considered significant for being the first year, allowed us to make suggestions for improvements; the main idea is to get the students learn to work with innovative methodologies.

This paper focuses on exposing the innovation process launched during the academic year 20132014 for the four bachelors taught at the Gandia Campus of Universitat Politècnica de Valencia. One of the main intentions about the commitment to develop these actions that drive innovation, generating new business and business ideas, is to promote entrepreneurship and intra entrepreneurship; given the bad economic situation, this is a way of improving economy also in general.

KEYWORDS: business creation, entrepreneurship, intra entrepreneurship, multidisciplinary, creativity, teamwork, innovation

\section{INTRODUCCIÓN}

La adaptación al Espacio Europeo de Educación Superior (EEES) implica un diseño de las asignaturas basado en competencias y objetivos de aprendizaje, que afectan tanto a la metodología de enseñanza-aprendizaje, como a la evaluación de los estudiantes. Tengamos en cuenta que los nuevos títulos de grado están estructurados en módulos o materias en cuyos objetivos de aprendizaje deben figurar ulas competencias genéricas y específicas que los estudiantes deben adquirir durante sus estudios y que sean exigibles para otorgar el título» tal y como figura en el Real Decreto del 29 de octubre de 2007.

Por otro lado, los egresados, al optar a un empleo, son evaluados no sólo por sus conocimientos técnicos sino en gran medida por las competencias personales que puedan aportar para el desempeño de sus tareas, donde la creatividad e innovación (Fayós et al., 2011) adquieren mayor presencia cada día, valorándose el emprendedurismo y el intraemprendedurismo. Existen diversos estudios sobre las competencias personales de los egresados universitarios para integrarse en la sociedad del conocimiento y sobre el papel que desempeñan las universidades en el desarrollo de dichas competencias, como Accenture y la plataforma Universia (2007); Hoffman (2003) y ANECA (2007). Estos estudios hacen que desde la universidad investiguemos cómo desarrollar esas competencias en los estudiantes y, por tanto, como evaluarlas. 


\section{QUÉ ENTENDEMOS POR COMPETENCIAS, CREATIVIDAD, INNOVACIÓN, EMPRENDEDURISMO E INTRAEMPRENDEDURSIMO}

La competencia, se resume por muchos autores en saber-hacer. Para Ardila (2004), existen variados usos para la expresión saber-hacer: uso creativo de vivencias y maneras de estar en el mundo, desempeños idóneos en la cotidianidad o como el dominio de una gramática básica Goñi (2005) define las competencias como la capacidad para enfrentarse con garantías de éxito a una tarea en un contexto determinado. Además, considera que la persona no es sólo capaz de realizar actividades mecánicas y repetitivas en el contexto en el que aprendió y que frente a la menor variación contextual se muestra incapaz de responder adecuadamente.

Si algo caracteriza a las sociedades actuales es la dificultad de prever todas las actividades que son necesarias para enfrentarse a los problemas que plantea el vivir, es difícil, porque son muchas y porque además cambian constantemente. Por esta razón, enunciar las competencias como capacidades es pertinente, Entendiendo de esta manera que el proceso instructivo es un proceso de conocimiento y no un proceso de acumulación (Goñi, 2005).

Siguiendo esto, las competencias son una combinación de atributos, habilidades y actitudes que se configuran como típicas del ejercicio de una profesión (jurídica, política, socióloga, técnica, etc.), que permiten una formación integral y que deben ser desarrolladas a lo largo del proceso de aprendizaje-enseñanza de los estudiantes a través de la aplicación de diferentes dinámicas. El estudiante debe tener una capacidad determinada, pero, además, saber ejercerla (Delgado et al., 2006).

En conexión con el concepto de competencia, Delgado et al. (2006) afirman que los resultados de aprendizaje son conjuntos de competencias que reflejan lo que el estudiante conocerá y será capaz de hacer al finalizar el proceso de aprendizaje. De ahí la importancia del diseño de cualquier asignatura en función de las competencias a las que debe contribuir, relacionando la metodología de enseñanza-aprendizaje con el resultado, que será conocer lo que el alumno es capaz de hacer a través de la metodología de evaluación (Santandrev et al., 2013).

Se llama creativa a una persona cuando de manera permanente obtiene resultados creativos, originales y prácticos. Halpern (1984) afirma que se puede definir como la habilidad de formar nuevas combinaciones de ideas para llenar una necesidad acuciante o resolver un problema y destaca una característica importante del pensamiento creativo: es un pensamiento estructurado, se puede aprender, de tal manera que tiende a llevarnos a resultados creativos. El criterio último de la creatividad es el resultado. Nosotros en la formación buscamos resultados; pues si la creatividad es tan demandada en la sociedad hoy en día como resultado enseñémosla y obtengamos resultados. Como dice el padre del pensamiento creativo, Bono (1995), el pensamiento creativo trata del cambio de conceptos y percepciones y de la generación de otros nuevos. Los productos finales son las ideas utilizables.

Para que los productos finales se transformen en ideas utilizables necesitamos el emprendedurismo y el intraemprendedurismo. Martínez (2003) considera dirección, supervisión y riesgo, como las funciones del empresario, y diferencia a este del emprendedor por el riesgo como el principal objetivo distintivo entre el emprendedor (entrepreneur) y el gerente. Sigue la definición del término intraemprendedor (intrapreneurship) considerándolo como un mecanismo (existente en las empresas) que puede también llenar el vacío entre la ciencia y el mercado. Según este autor, las empresas que poseen recursos financieros, habilidad administrativa y sistemas de 
distribución y marketing para comercializar exitosamente sus productos, poseen frecuentemente una estructura burocrática, el énfasis en beneficios a corto plazo y una gran estructura organizativa que inhibela creatividad y el desarrollo de nuevos productos. Así mismo, las empresas que reconocen estas limitaciones y la necesidad de creatividad e invención, intentan establecer un espíritu intraemprendedor en sus organizaciones; es decir, emprendimiento dentro de una estructura administrativa existente. Enseñemos a nuestros alumnos desde la educación superior a trabajar así ya porque independientemente de su formación cuando se incorporen al mundo laboral solo tiene dos vías: una, montar una empresa, o dos, trabajar en una empresa. En cualquier caso su fin, su objetivo, su resultado, es la empresa.

Las competencias emprendedoras son el conjunto de conocimientos, habilidades y actitudes que le permiten al estudiante canalizar sus ideas e intereses en el marco de las tendencias cambiantes del entorno (Santandreu, 2013), basado en los conocimientos adquiridos, con el fin de formular proyectos que contribuyan a su propio desarrollo y al del entorno, teniendo en cuenta los criterios de relevancia y viabilidad (Gómez y Jacobsohn, 2007).

La importancia de articular el emprendimiento con los planes de estudio es un tema que actualmente se está tratando de implementar en la mayoría de las universidades. Afirman Gómez y Jacobsohn (2007) que las universidades, al incorporar temas de emprendimiento en el currículo de los programas profesionales, buscan los siguientes objetivos: incidir en el crecimiento económico de la comunidad; cubrir una demanda cada vez mayor de estudiantes que buscan crear sus propias organizaciones; aumentar la reputación para atraer a nuevos estudiantes de pregrado y postgrado; desarrollar un entorno económico y social más sano formando emprendedores con alta responsabilidad social; y reforzar la cultura emprendedora de su comunidad o país. En este contexto, se busca generar y desarrollar actitudes, conocimientos y habilidades para emprender exitosamente.

Las habilidades emprendedoras son el complemento de los conocimientos necesarios para crear y administrar nuevas empresas o mejorar las ya existentes. El desarrollo de las asignaturas debe ofrecer a los estudiantes la posibilidad de que desarrollen habilidades y competencias genéricas y específicas acordes con el sector empresarial (Canós, et al., 2011).

Según Gómez y Jacobsohn (2007), ello es posible si al estudiante se le confronta con situaciones de negocios reales y complejos, al resolver casos, al desarrollar planes de negocios y al poner en marcha actividades, negocios, empresas $u$ organizaciones. En consecuencia, una educación basada en competencias se define como la convergencia entre los conocimientos de las disciplinas, las habilidades genéricas y la comunicación de las ideas. La simple aceptación del desarrollo de competencias como propósito central de la educación implica, como ya se ha sugerido, la reorientación de las prácticas de enseñanza, específicamente, la revisión, selección y organización cuidadosa de los contenidos y actividades curriculares (Bogoya et al., 2000).

\section{DISEÑO DE LA ASIGNATURA CREACIÓN DE EMPRESAS}

Nuestro propósito se ha basado en diseñar una asignatura optativa para alumnos de cuarto curso, Creación de Empresas, que se oferta a los cuatro grados que se imparten en la Universitat Politècnica de Valencia en el Campus de Gandía. Dichos grados son el grado en gestión turística (GGT), el grado en comunicación audiovisual (GGCA), el grado en ciencias ambientales (GCCAA) y el grado en ingeniería de sistemas de telecomunicación, sonido e imagen (GISTSI). Los alumnos que asisten 
Santandreu-Mascarell, C; Canós-Darós, L. y Marin- Roig, J.R.

Fomentando la multidisciplinariedad y el trabajo en equipo (metodologías participativas)

durante el semestre A pertenecen a GCCAA y GGT; en el semestre B, GGCA y GISTSI. Esta agrupación ha sido por motivos académicos de horarios. Nuestra intención era poder unir a los cuatro grados, con el objetivo de poder conseguir mediante la diversidad en la formación y experiencias una mayor creatividad y al mismo tiempo que los estudiantes formando grupo multidisciplinares trabajen como en el ámbito profesional, ya que en este no decidirán ni a sus compañeros, ni su formación y experiencias.

Las razones que justifican la elección de crear y ofertar esta nueva asignatura aplicando metodologías de creatividad e innovación se pueden sintetizar, expuesto todo lo anterior, en:

- Adecuar nuestra acción docente a los planteamientos metodológicos y de evaluación pautados por el Espacio Europeo de Educación Superior (EEES): competencias genéricas que son comunes a las cuatro profesiones y que se relacionan con la puesta en práctica integrada de aptitudes, rasgos de personalidad, conocimientos y valores adquiridos.

- $\quad$ Adecuar nuestra acción docente a la demanda profesional: competencias que permitan innovar y permitan desenvolverse en el ámbito profesional, la empresa.

Teniendo en cuenta los resultados que se pretenden alcanzar y siguiendo y adaptando el esquema de la creatividad, Iglesias y Rodicio (2013), se presenta el diseño de la asignatura, Creación de Empresas, en la Tabla 1. Esquema creatividadDiseño: Creación de Empresas.

TABLA 1. ESQUema CREATIVIDAD-DISEÑo: Creación De EMPRESAS.

\begin{tabular}{|l|l|}
\hline Esquema creatividad & Diseño: Creación de Empresas \\
\hline $\begin{array}{l}1^{\circ} \text { Actitud y aptitud creativa: la aptitud se } \\
\text { entrena }\end{array}$ & $\begin{array}{l}1^{\circ} \text { Selección de metodologías de enseñanza- } \\
\text { aprendizaje }\end{array}$ \\
\hline $2^{\circ}$ Proceso creativo: técnicas y métodos & $\begin{array}{l}2^{\circ} \text { Selección de metodologías para fomentar el } \\
\text { proceso creativo }\end{array}$ \\
\hline $\begin{array}{l}3^{\circ} \text { Producto resultante: ideas, cosas nuevas, } \\
\text { innovaciones }\end{array}$ & $3^{\circ}$ Selección de técnicas de evaluación \\
\hline $4^{\circ}$ Sociocultural: expertos que evalúan & $4^{\circ}$ Resultados: expertos que evalúan \\
\hline
\end{tabular}

La actitud creativa es propia de la persona, está formada por el instinto de curiosidad, el inconformismo, la motivación, la iniciativa, la profundidad, la perseverancia y la autoestima, mientras que las aptitudes se pueden aprender y entrenar, existen técnicas y métodos para ello. En esta parte es donde entramos nosotros con la selección de técnicas para fomentar la creatividad dentro de las metodologías de enseñanza-aprendizaje.

Las unidades didácticas, son el conjunto organizado, integrado, secuencial y estructurado de objetivos, contenidos, metodologías, actividades y recursos didácticos, que tienen sentido por sí mismos y que facilitan a los estudiantes el aprendizaje (Guía docente de la UPV: criterios para su elaboración). Las unidades docentes en que se distribuye la asignatura son las que se muestran en la Figura 1. Unidades didácticas. 


\section{FIGURA 1. UNIDADES DIDÁCTICAS}

\section{Unidades Didácticas:}

1. Generación de ideas: creatividad/innovación. Visual Thinking.

1. Formando equipos de trabajo.

2. Definiendo los desafíos.

3. Herramientas de creatividad lineal.

4. Herramientas de creatividad intuitiva.

5. Gestión de ideas.

2. Modelo de negocio y modelo de operaciones.

1. Resumen ejecutivo.

2. Descripción del proyecto.

3. Mercado (Mapa de empatía y Curvas de Valor).

4. Valor diferencial y ventajas competitivas (Business modelCanvas y Business modeltoolbox).

5. Equipo.

6. Estrategia y cadena de valor (Business modelCanvas y Business modeltoolbox).

7. Análisis de la situación (DAFO).

8. Momento actual-Hitos.

9. Aspectos económico-financieros.

10. Riesgos y otros.

\section{Presentación.}

1. Elevator Pitch (1 minutos).

2. Presentación formal (20 Minutos).

El proceso creativo es la aplicación de reglas técnicas y métodos; en nuestro caso son las que se muestran en la Tabla 2. Unidades-Técnicas creativas. Se han seleccionado en función de los objetivos formativos a alcanzar con cada unidad.

TABLA 2. UNIDADES-TÉCNICAS CREATIVAS.

\begin{tabular}{|l|l|}
\hline Unidades & Técnicas creativas: proceso creativo \\
\hline Generación de ideas & 1-Mapa de contexto. \\
& 2-Desafio personal. \\
& 3-Desafio del grupo. \\
& 4-Palabras al azar. \\
& 5-Las profesiones. \\
\hline Modelo de negocio & 1-Mapa de empatía. \\
& 2-Curva de valor. \\
& 3-Business Model Canvas. \\
& 4-Business Model Toolkit. \\
& 5-Lego \\
\hline Presentación & 1-Elevator Pitch. \\
& 2-Defensa de tu plan de negocio ante un grupo de expertos \\
\hline
\end{tabular}

El objetivo de la asignatura: los alumnos, a través de la gestión del conocimiento y el trabajo en equipo multidisciplinar deben ser capaces de generar una idea de negocio, mediante distintas técnicas de creatividad, evaluar y valorar esa idea de negocio para ver si es factible y plasmarla en un plan de negocio. Para poder trabajar este fin, el objetivo se desglosa en unidades.

Primera unidad: Generación de ideas. El objetivo es aplicar técnicas creativas para que el alumno pueda dar paso a sus actitudes y aptitudes creativas dando lugar a diversas ideas de negocio, productos, servicios, etc.

Segunda unidad: modelo de negocio. El objetivo es centrar una de las ideas y plasmarla en un plan de negocio, no convencional, al que vamos a llegar a su elaboración mediante la utilización de las técnicas creativas que se muestran en la 
Tabla 2. Unidades-Técnicas creativas, esperando conseguir el producto resultante que es la consecución de algo nuevo e innovador que se puede aplicar en la dimensión sociocultural y que será los expertos de esta quien lo evalúe.

En la tercera unidad: presentación. El objetivo es que los alumnos muestren sus resultados. Para ello deben presentar en un portafolio los resultados obtenidos con cada técnica creativa de forma individual, y además, presentar y defender un plan de negocio para que sea evaluado el trabajo en grupo y la exposición en grupo e individual.

Teniendo en cuenta todo esto, se elabora la guía docente de la asignatura, que consta de las siguientes partes, entrando solo en las partes relevantes para este trabajo que son:

1. Datos identificativos.

2. Competencias específicas y genéricas.

3. Conocimientos recomendados.

4. Selección y agrupación de contenidos.

5. Metodologías.

6. Evaluación.

7. Recursos y bibliografía.

De las metodologías de enseñanza-aprendizaje, consultadas en la web de la Universitat Politècnica de Valencia, se opta por (teniendo en cuenta las competencias a las que contribuir en cada grado y las demandadas en el ámbito profesional, los objetivos de las unidades y el sistema de evaluación) las actividades de carácter presencial y de trabajo autónomo que se muestran en las tablas 3 y 4 , respectivamente.

TABLA 3. ACtividAdes de CARÁCter presencial.

\begin{tabular}{|c|c|}
\hline \multicolumn{2}{|c|}{ ACTIVIDADES DE TRABAJO PRESENCIAL } \\
\hline Clase presencial & $\begin{array}{l}\text { Exposición de contenidos mediante presentación o explicación } \\
\text { por parte de un profesor (posiblemente incluyendo } \\
\text { demostraciones). }\end{array}$ \\
\hline Trabajo en grupo & $\begin{array}{l}\text { Sesión supervisada donde los estudiantes trabajan en grupo y } \\
\text { reciben asistencia y guía cuando es necesaria. }\end{array}$ \\
\hline Caso & $\begin{array}{l}\text { Técnica en la que los alumnos analizan situaciones profesionales } \\
\text { presentadas por el profesor, con el fin de realizar una } \\
\text { conceptualización experiencial y realizar unabúsqueda de } \\
\text { soluciones eficaces. }\end{array}$ \\
\hline $\begin{array}{l}\text { Aprendizaje basado en } \\
\text { proyectos }\end{array}$ & $\begin{array}{l}\text { Situaciones en las que el alumno debe explorar y trabajar un } \\
\text { problema práctico aplicando conocimientos interdisciplinares. }\end{array}$ \\
\hline $\begin{array}{l}\text { Presentación de trabajos } \\
\text { de grupo }\end{array}$ & $\begin{array}{l}\text { Exposición de ejercicios asignados a un grupo de estudiantes que } \\
\text { necesita trabajo cooperativo para su conclusión. }\end{array}$ \\
\hline Laboratorio & $\begin{array}{llll}\text { Actividades desarrolladas en espacios especiales } & \text { con } \\
\text { equipamiento especializado (laboratorio, aulas informáticas). } & \end{array}$ \\
\hline
\end{tabular}

FUENTE: WWW.UPV.ES. 
TABla 4. Actividades de trabajo autónomo.

\begin{tabular}{|l|l|}
\hline ACTIVIDADES DE TRABAO AUTÓNOMO \\
\hline Estudio teórico & $\begin{array}{l}\text { Estudio de contenidos relacionados con las clases teóricas: Incluye } \\
\text { cualquier actividad de estudio que no se haya computado en el apartado } \\
\text { anterior (estudiar exámenes, trabajo en biblioteca, lecturas } \\
\text { complementarias, hacer problemas y ejercicios, etc.). }\end{array}$ \\
\hline Estudio práctico & Relacionado con las clases prácticas. \\
\hline
\end{tabular}

FUENTE: WWW.UPV.ES.

Las técnicas de evaluación seleccionadas son las que se muestran en la Tabla 5. Técnicas de evaluación:

TABLA 5. TÉCNICAS de eVAlUACIÓN. Fuente: WWW.uPV.es.

\begin{tabular}{|l|l|}
\hline $\begin{array}{l}\text { Mapa conceptual: } \\
\text { Individual }\end{array}$ & $\begin{array}{l}\text { Muestra la forma de relacionar los conceptos clave de un área } \\
\text { temática. }\end{array}$ \\
\hline $\begin{array}{l}\text { Portafolio: descripción } \\
\text { individual de las sesiones. }\end{array}$ & $\begin{array}{l}\text { Conjunto documental elaborado por un estudiante que muestra } \\
\text { la tarea realizada durante el curso en una materia determinada. }\end{array}$ \\
\hline $\begin{array}{l}\text { Proyecto: plan de negocio. } \\
\text { Grupo }\end{array}$ & $\begin{array}{l}\text { Es una estrategia didáctica en la que los estudiantes desarrollan } \\
\text { un producto nuevo y único mediante la realización de una serie } \\
\text { de tareas y el uso efectivo de recursos. }\end{array}$ \\
\hline Caso: en grupo & $\begin{array}{l}\text { Supone el análisis y la resolución de una situación planteada que } \\
\text { presenta problemas de solución múltiple, a través de la reflexión y } \\
\text { el diálogo para un aprendizaje grupal, integrado y significativo. }\end{array}$ \\
\hline
\end{tabular}

Se observa una relación clara entre las metodologías de enseñanza-aprendizaje y el sistema de evaluación, ya que no se puede evaluar algo que no se enseña-aprende:

1-Un portafolio en el que se muestre las tareas realizadas durante el curso. 10\%

2-Mapa conceptual que plasme la fase de creatividad. Generación de ideas. 20\%

3-Herramientas visuales plan de negocio. $30 \%$

4-Un proyecto final. Se divide en dos partes: plan de negocio $30 \%$ y presentación y defensa del proyecto mediante herramientas visuales $10 \%$.

\section{IMPLANTACIÓN Y DESARROLLO DE LAS CLASES}

El objetivo de la asignatura es, a través de la práctica, llegar a la teoría mediante su identificación, aprendizaje y aplicación. Las sesiones de las clases están preparadas para combinar las técnicas de creatividad con los contenidos de cada unidad. Primero, se realiza la técnica de creatividad selecciona acorde con el objetivo de aprendizaje y seguidamente se identifica éste objetivo de aprendizaje conceptualmente, permitiendo esta combinación en el proceso de enseñanzaaprendizaje abarcar los conceptos, aptitudes y actitudes En la elaboración y preparación de las sesiones los profesores han tenido en cuenta que los alumnos aprendan y trabajen con las técnicas de evaluación que se les iba a exigir como se observa en la Figura 1. Unidades didácticas. 
Santandreu-Mascarell, C; Canós-Darós, L. y Marin- Roig, J.R.

Fomentando la multidisciplinariedad y el trabajo en equipo (metodologías participativas)

El trabajo práctico realizado durante las sesiones por los alumnos lo plasman en el portafolio y en el proyecto dando lugar mediante el aspecto formal a poder evaluar el profesor si han alcanzado las competencias y pudiendo los alumnos llevar la asignatura prácticamente al día.

Para conseguir el objetivo la metodología de trabajo que se lleva a cabo es la de formar grupos multidisciplinares que combinen alumnos de los distintos grados. Además se invita a personas de fuera de estos grados (alumnos de máster, emprendedores, empresarios, etc.) a participar en la asignatura para conseguir un mayor aporte de ideas y creatividad. Con esto los grupos quedan formados para trabajar todo el cuatrimestre.

Otro aspecto importante para alcanzar el objetivo de la asignatura es su control y la distribución de las unidades por sesiones. Por dos motivos: uno, tenemos limitación temporal para impartir la asignatura y dos, las técnicas de creatividad deben ser controladas temporalmente para conseguir unos resultados eficaces y eficientes y además, en nuestro caso las combinamos y llevamos a los contenidos teóricos para su aplicación.

La asignatura tiene asignada 15 sesiones de 210 minutos, en el semestre A, lo que implica un gran esfuerzo para el docente, debe diseñar cada sesión para sacar el máximo partido del alumno teniendo en cuenta, la capacidad de retención. La distribución que se llevó a cabo y que se cumplió prácticamente se muestra en la Tabla 6. Distribución sesiones.

TABLA 6. DISTRIBUCIÓN SESIONES.

\begin{tabular}{|c|c|}
\hline \multicolumn{2}{|l|}{ SEPTIEMBRE } \\
\hline VIERNES 6: Presentación de la asignatura. & VIERNES 13: Unidad 1 y formar equipos. \\
\hline VIERNES 20: Unidad 1. & VIERNES 27: Unidad 1 \\
\hline \multicolumn{2}{|l|}{ OCTUBRE } \\
\hline VIERNES 4: Unidad 1. & VIERNES 11: Unidad 1. \\
\hline VIERNES 18: Unidad 1. & VIERNES 25: Unidad 2. \\
\hline \multicolumn{2}{|l|}{ JUEVES 31: Unidad 2.} \\
\hline \multicolumn{2}{|l|}{ NOVIEMBRE } \\
\hline VIERNES 8: Unidad 2. & VIERNES 15: Unidad 2. \\
\hline VIERNES 22: Unidad 2. & VIERNES 29: Unidad 2. \\
\hline \multicolumn{2}{|l|}{ DICIEMBRE } \\
\hline VIERNES 13: Unidad 2. & VIERNES 20: Unidad 3. \\
\hline \multicolumn{2}{|c|}{$\begin{array}{l}\text { PRESENTACIÓN / EXAMEN : } 13 \text { ENERO } \\
\text { Exposición del plan de negocio: en grupo (presentación formal) e individual (elevator } \\
\text { pitch) }\end{array}$} \\
\hline
\end{tabular}

En el semestre B, se siguió el mismo criterio.

\section{RESULTADOS DE LOS TRABAJOS DE LOS ALUMNOS}

Ante la dificultad de explicar y transmitir de forma clara esta forma de trabajar con los alumnos, se muestra a continuación resultados de alguna de las sesiones.

Durante la sesión 2 se forman los grupos. Estos se ponen a trabajar en la realización de un mapa conceptual. El resultado de esta tarea se muestra en las Fotos 1. Mapa conceptual: 
Santandreu-Mascarell, C; Canós-Darós, L. y Marin- Roig, J.R. Fomentando la multidisciplinariedad y el trabajo en equipo (metodologías participativas)

FOTOS 1. MAPA CONCEPTUAL

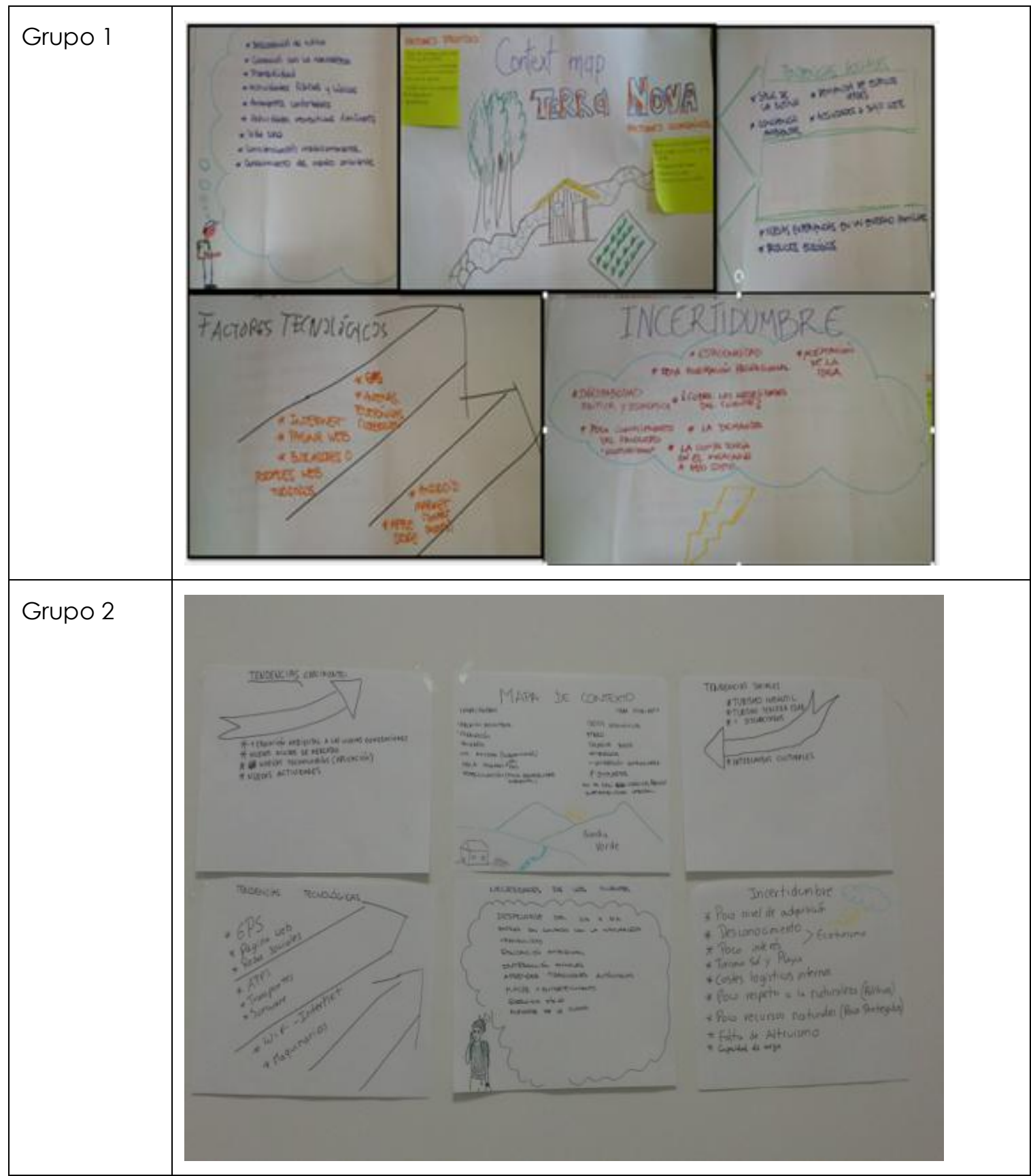

El mapa conceptual permitió situar a los alumnos en una temática en concreto, describiendo la situación actual en la que nos encontramos para montar una empresa.

Siguiendo la distribución de sesiones mostrada en la Tabla 6 y la combinación de técnicas creativas con las unidades mostrada en la Tabla 2. Se presentan, en las Fotos 2. Técnicas de creatividad y generación de ideas, los resultados del grupo 1 de la aplicación de una técnica de creatividad, palabras al azar y los resultados del grupo 2 , realización de un mapa de empatía. 


\section{FOTOS 2. TÉCNICAS DE CREATIVIDAD Y GENERACIÓN DE IDEAS}

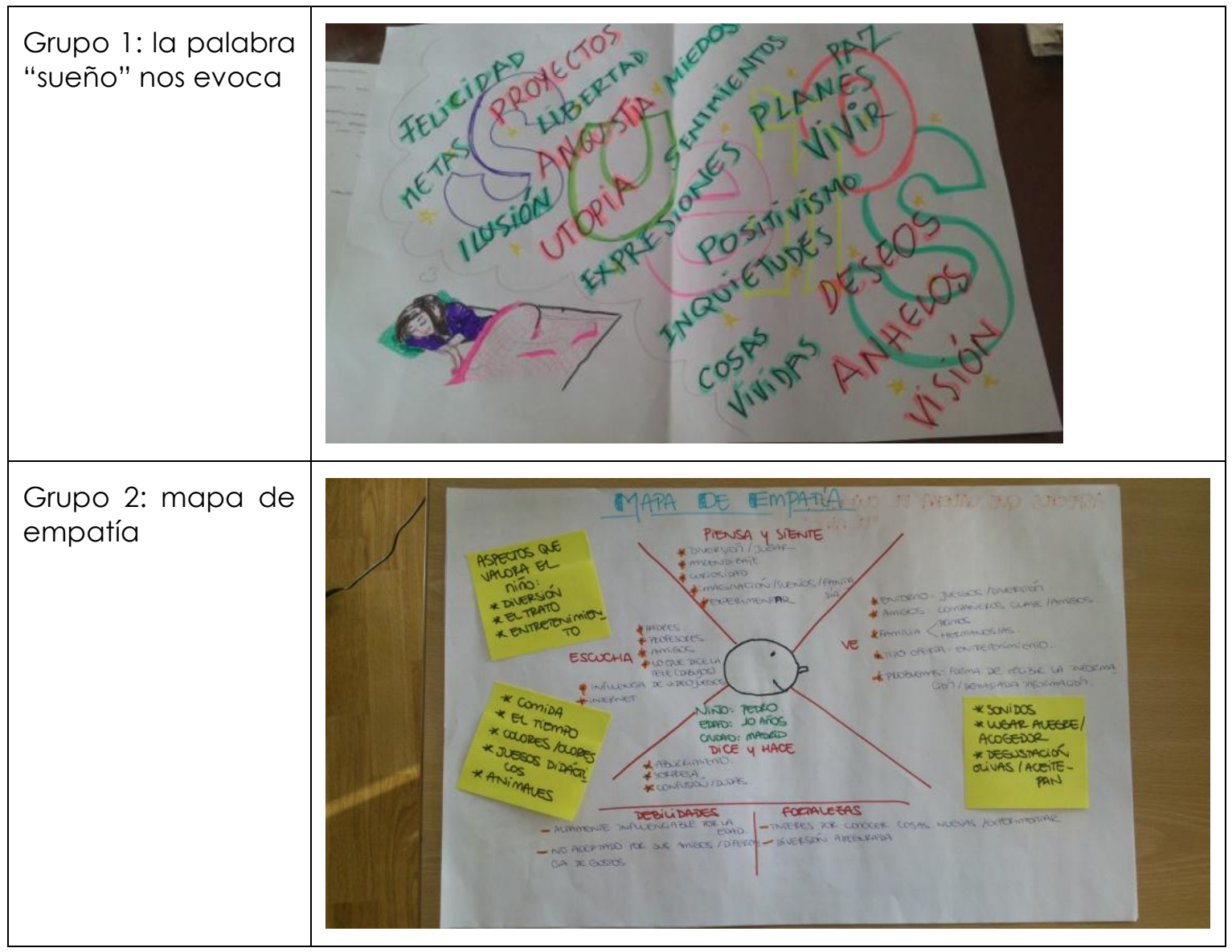

Con estas técnicas, los alumnos consiguen generar distintas ideas de planes de negocio y seleccionar una de ellas para continuar trabajando. Así el grupo 1 decide estudiar la posibilidad de crear una aldea rural y el grupo 2, una empresa de aceite especializado para la alta hostelería de forma que sus instalaciones puedan ser visitadas como reclamo turístico.

Una vez llegado a este punto, se realiza con ellos el Business Model Canvas, Business Model Toolbox y el Lego, correspondientes al aprendizaje-enseñanza de la unidad 2. Esto les va a permitir de una forma creativa poder llevar a cabo cada uno de los puntos del plan de negocio que deben entregar por grupos. Podemos ver los resultados en las Fotos 3. Business Model Canvas Business Model Toolbox-Lego. 
Santandreu-Mascarell, C; Canós-Darós, L. y Marin- Roig, J.R. Fomentando la multidisciplinariedad y el trabajo en equipo (metodologías participativas)

Fotos 3. Business Model Canvas-Business Model ToolboX-Lego

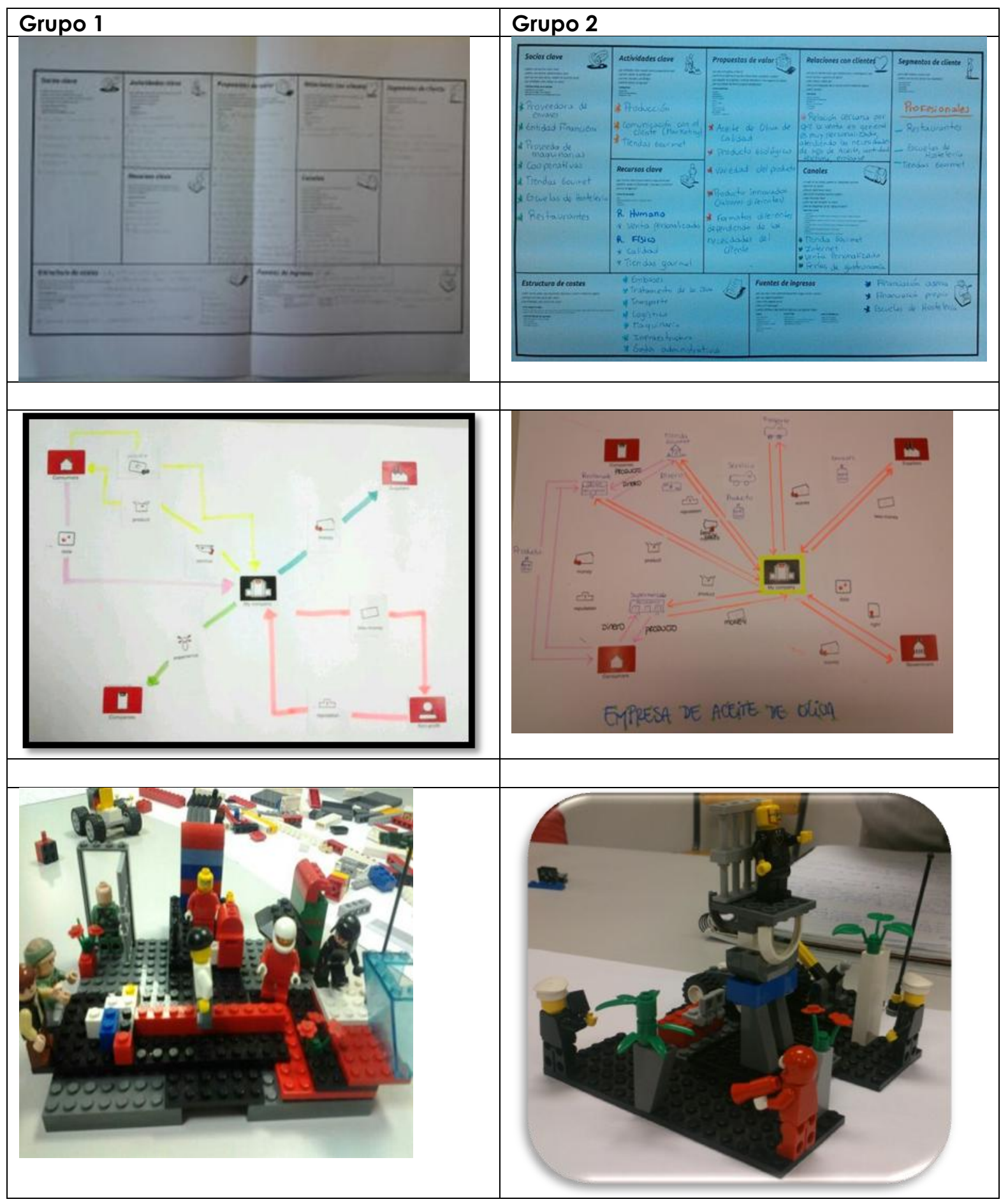

\section{RESULTADOS OBTENIDOS. CALIFICACIONES DE LOS ALUMNOS}

Como se ha comentado anteriormente se evalúa lo que se enseña, así nuestro sistema y resultados de la evaluación del semestre A, fueron los que se muestran en la Tabla 7. Resultados semestre A: 
Santandreu-Mascarell, C; Canós-Darós, L. y Marin- Roig, J.R.

Fomentando la multidisciplinariedad y el trabajo en equipo (metodologías participativas)

\begin{tabular}{|c|c|c|c|c|c|c|c|}
\hline & $\frac{\text { Portaflio } 10 \%}{\text { (1 PUNTO) }}$ & $\frac{\text { Herramientas }}{\text { visuales }}$ & $\frac{\text { Mapa conceptual }}{20 \%}$ & \multicolumn{4}{|c|}{ Plan de negocio $40 \%$ (4 PUNTOS) } \\
\hline & & $\begin{array}{l}30 \%(3 \\
\text { puntos) }\end{array}$ & & $\begin{array}{c}\text { Plan de } \\
\text { negocio } 3\end{array}$ & \multicolumn{2}{|c|}{ Presentaciones 1} & \\
\hline Alumno & & & & & $\begin{array}{c}\text { Grupo } \\
0,7\end{array}$ & Indv 0,3 & \\
\hline Turismo & 0,8 & 2,6 & 2 & 2,8 & 0,7 & 0,18 & 9,08 \\
\hline Turismo & 0,5 & 1,7 & 2 & 2,8 & 0,7 & 0,3 & 8 \\
\hline Ambientales & 0,7 & 3 & 2 & 2,8 & 0,7 & 0,3 & 9,5 \\
\hline Turismo & 0,4 & 1,2 & 2 & 1,8 & 0,28 & 0,18 & 5,86 \\
\hline Ambientales & 0,6 & 1,8 & 2 & 1,8 & 0,28 & 0,15 & 6,63 \\
\hline Turismo & 0,7 & 3 & 2 & 1,8 & 0,28 & 0 & 7,78 \\
\hline Ambientales & 0,2 & 0,6 & 2 & 1,2 & 0,28 & 0,9 & 5,18 \\
\hline Turismo & 0,5 & 1,5 & 2 & 2,8 & 0,7 & 0,24 & 7,74 \\
\hline
\end{tabular}

Como se puede observar en la tabla 7 , se evalúo según el proceso de enseñanzaaprendizaje seleccionado.

Se consiguió un $100 \%$ de aprobados. Tres sobresalientes, 2 notables y 3 aprobados.

Tras esta primera experiencia, se realizaron cambios en el sistema de evaluación, ya que se realizaron cambios para facilitar el proceso de enseñanza -aprendizaje para mejorar esta y mejorar la formación del alumno.

Por eso la Tabla 8. Resultados semestre B es distinta de la Tabla 7. Resultados semestre A:

Como se puede observar con ambas tablas (7 y 8), los cambios que se realizaron fueran más de organización y concreción que de materia, se reestructuró la forma de impartir las unidades y las relaciones entre ellas, siendo el mismo contenido y siguiendo la misma metodología y además, se concretó mejor el sistema de evaluación, con el fin de que el alumno conociera con más detalle que se le pedía en su evaluación, de qué se le iba a evaluar de todo aquello que estaba aprendiendo.

En esta tabla se puede observar los resultados de cada alumno y ver a qué grupo han pertenecido cada uno y comparar los resultados obtenido entre ellos.

De los 17 alumnos que cursaron la asignatura en el semestre B, solo suspendieron 2, y como se observa en la Tabla 8, por falta de trabajo individual. No hay ningún sobresaliente, la mayoría son notables o mejor dicho la totalidad de estos son prácticamente notables.

Al ser el primer curso no podemos mostrar ninguna evidencia significa de mejora de evaluación de la experiencia, ni vía incremento de tasas de rendimiento. Pero si que hay algo que avala nuestro trabajo y es la matrícula del curso 2014-2015 que se ha incrementado notablemente, siendo en el semestre A de 25 alumnos y en el semestre $B$ de 22, frente a los 8 y 17, respectivamente del curso 2013-2014, se supone que el "boca-oreja" ha sido bueno. 
Santandreu-Mascarell, C; Canós-Darós, L. y Marin- Roig, J.R. Fomentando la multidisciplinariedad y el trabajo en equipo (metodologías participativas)

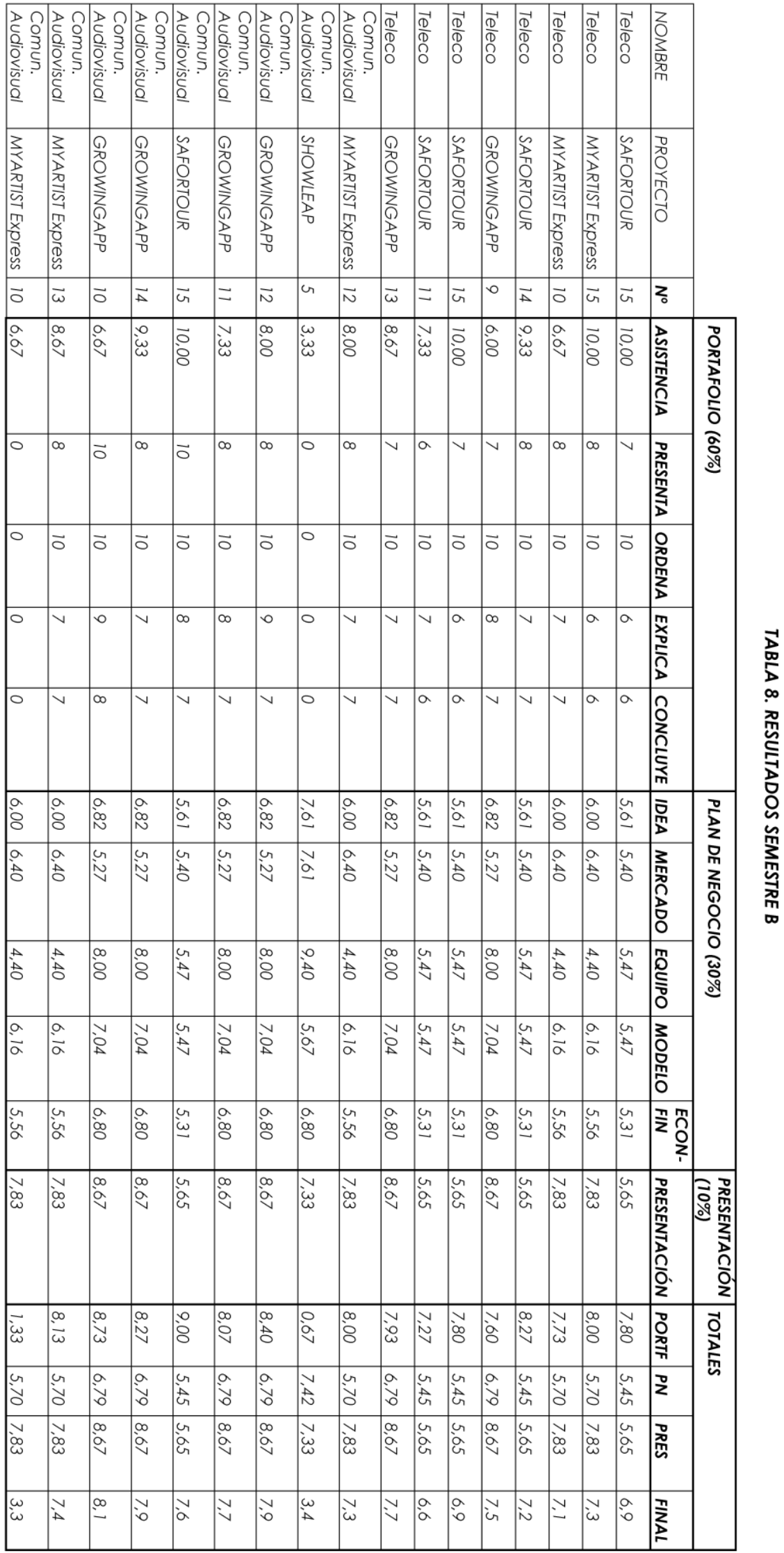

educade, no 6, 2015, p. 94 


\section{CONCLUSIONES}

Los proyectos emprendidos nos permiten avanzar dando respuesta a las incertidumbres sobre la puesta en práctica de una formación adaptada a EEES y al ámbito profesional.

El hecho de reflexionar juntos, dialogar sobre lo que hacemos y afrontarlos obstáculos que se presentan día a día en nuestras aulas nos enriquece como docentes al compartirlo con los demás. El trabajo en equipo y la reflexión compartida del grupo de profesorado han hecho más fácil el generar algo diferente e innovador. Se trata de establecer un compromiso de desarrollo profesional para la mejora de la calidad en la docencia universitaria.

En el proceso de innovación aquí presentado, el centro de interés de nuestra propuesta se ha basado en la metodología participativa y la evaluación formativa que se ponen en marcha en los nuevos títulos europeos de grado.

El uso de instrumentos alternativos a lo tradicional, como puede ser el portafolio, las técnicas creativas; pasar de la práctica a la teoría, han permitido mejorar la calidad de la participación del alumnado en las actividades individuales y grupales de la asignatura, generando cambios significativos en la forma de entender la enseñanza por el profesorado y el alumnado.

Se necesitan cambios que debemos ir articulando progresivamente, aunque en ocasiones suponga luchar con las tradiciones educativas y fomentar el cambio de paradigma desde la reflexión.

Por ahora y teniendo en cuenta que la asignatura durante el curso 2013-2014 es la primera vez que se imparte, según los resultados y la observación de los profesores que han vivido la experiencia, nos quedamos para proponer y establecer mejoras en el curso 2014-2015, con:

- hay que reajustar la metodología de enseñanza-aprendizaje y el sistema de evaluación para formar al alumno en un trabajo más participativo (en grupo) y con resultados en grupo e individuales, como ocurre en las empresas, con una metodología por parte del alumno más autónoma y responsable.

- hay que tener en cuenta la diferencia de formación en los distintos grados, se notó mucho a su favor, en este caso, que los alumnos del Grado en Gestión Turística les resultaba mucho más fácil y estaban mucho más cómodos con esta metodología.

Como afirma Berge: Educar a un niño es esencialmente enseñarle a prescindir de nosotros.

\section{BIBLIOGRAFÍA}

ACCENTURE Y UNIVERSIA (2007): Competencias profesionales en los titulados. Contraste y diálogo Universidad-Empresa.

ANECA: Jornadas REFLEX, Informe ejecutivo: El profesional flexible en la Sociedad del Conocimiento. CEGES V. $2.0 \quad-\quad 28 / 06 / 2007 . \quad$ Disponible enhttp://www.aneca.es/estudios/docs/InformeejecutivoANECA_jornadasREFLEXV20.pd f.

ARDILA, L.F (2004): El concepto de competencias v2. Una mirada interdisciplinar. Editorial Alejandría Libros. 
BOGOYA, D., VINENT, M., RESTREPO, G., TORRADO, C.M., JURADO, F., PÉREZ, M., ACEVEDO, M., GRACÍA, G., SARMIENTO, F., CÁRDENAS, F., GRANÉS, J. Y DÍAZ, L.G (2000): Competencias y proyecto pedagógico. Santafé de Bogotá, Universidad Nacional.

BONO, E (1995): El pensamiento creativo. Paidós Iberica. Barcelona.

CANÓS, L., PONS, C., Y SANTANDREU, C. (2011): Competencias que ofrecen las universidades españolas que imparten el Grado en Ingeniería en Tecnologías Industriales Grado en Ingeniería en Tecnologías Industriales. Working Papers on Operations Management 2, 1, 31-38.

CHRISTENSEN, C. M. (2011): The innovator's dilemma: the revolutionary book that will change the way you do business. Harper Business Essentials

DELGADO, A., BORGE B., GARCÍA A., OLIVER C., Y SANCHO S (2006): Competencias y diseño de la evaluación continua y final en el espacio europeo de educación superior. J.M. Bosh Editor. Barcelona.

FAYÓS, T., GONZÁlEZ-GALLARZA, M., SERVERA, D. Y ARTEAGA, F. (2011): Análisis y evaluación del servicio de formación universitaria: implicaciones para el marketing estratégico de las universidades. Revista de Investigación en Educación, 9, 133-152.

GÓMEZ, L. Y JACOBSOHN, G. (2007): Desarrollo de competencias emprendedoras.

GOÑI, J. (2005): El espacio europeo de educación superior, un reto para la universidad. Editorial Octaedro, S.L, Barcelona.

GOXE, F Y VIALA, C. (2001): Intraemprersario y movilización del personal: hacia una dinámica de innovación. Primer Congreso Internacional en México sobre la MIPYME: El impacto de la investigación académica en el desarrollo de la MIPYME.

Disponible en http://basepub.duphine.fr/xmlui/handle/123456789/9236.

GRAY D. (2012): Gamestorming: 83 juegos para innovadores, inconformistas y generadores del cambio. Grupo planeta Spain.

HALPERN, D. (1984): Thought and knowledge: an introuction to critical thinking. L. Erlbaum Assoc.

HOFFMAN,T. (2003): Preparing generationZ. ComputerWorld,

Disponible enhttp://www.computerworld.com/s/article/84295/Preparing_Generation_Z

IGLESIAS, M.J Y RODICIO, M.L. (2013): El desarrollo de la creatividad e innovación. Unreto ante la crisis actual. Revista de Investigación en Educación, n 11 (1), pp. 134148.

KAWASAKI, G. (2007): El arte de empezar: una guía infalible para empezar cualquier cosa. Ediciones Kantolla S.I. llustrae.

KIM, W. (2008): La estrategia del océano azul: cómo crear en el mercado espacios no disputados en los que la competencia sea irrelevante. Granica.

MARTíNEZ, P. (2003): Función Empresarial y Creación de Empresas «Entrepreneurship», como Programa de Investigación Científica. Corporación Universitaria del Caribe. (CECAR), Colombia Revista Búsqueda de CECAR No. 6.

MICHALKO, M. (2000): Thinkertoys: cómo desarrollar la creatividad en la empresa. Barcelona, Ediciones Gestión.

PRIETO, F.H. (2010): Competencies for entrepreneurship in the Business Administration Program at Corporación Universitaria del Caribe - CECAR. 
Santandreu-Mascarell, C; Canós-Darós, L. y Marin- Roig, J.R.

Fomentando la multidisciplinariedad y el trabajo en equipo (metodologías participativas)

OSTERWALDER, A. (2011): Generación de modelos de negocio: un manual para visionarios, revolucionarios y retadores. Deusto.

Real Decreto 1393/2007 del 29 de octubre de 2007 por el que se establece la ordenación de las enseñanzas universitarias oficiales. BOE núm. 260, pp. 44037 y ss. Martes 30 de octubre de 2007.

RIES, E. (2012): El método Lean Startup: cómo crear empresas de éxito utilizando la innovación continua. Grupo planeta.

SANTANDREU-MASCARELL, C., CANÓS-DARÓS, L., PONS-MORERA, C. (2012): Real companies demand of competences in higher education study plans. WSEAS Transactions on Advances in Engineering Education, Issue 1, 9, pp 23-30.

SANTANDREU, C., GARZÓN, D. Y KNORR, H. (2013): Entrepreneurial and innovative competences, are the same? Management Decision, 51, 5, pp 1084-1095 